\title{
Multidisciplinary quality improvement initiative to optimize acute neurovascular imaging for transient ischemic attack or minor stroke
}

\author{
Arunima Kapoor ${ }^{1} \cdot$ Aikta Verma $^{2} \cdot$ Isabelle J. Kim ${ }^{1} \cdot$ Nastasia Kujbid $^{1} \cdot K^{6 e v i n ~} \mathrm{Si}^{1} \cdot$ Leanne K. Casaubon $^{3}$. \\ Moira K. Kapral ${ }^{4,6}$. Jiming Fang ${ }^{6} \cdot$ Sean Symons ${ }^{1,5} \cdot$ Richard H. Swartz $^{1,6,7} \cdot$ Amy Y. X. Yu ${ }^{1,6,7}$ (])
}

Received: 20 March 2021 / Accepted: 19 July 2021 / Published online: 13 September 2021

(c) The Author(s), under exclusive licence to Canadian Association of Emergency Physicians (CAEP)/ Association Canadienne de Médecine d'Urgence (ACMU) 2021

\begin{abstract}
Background Neurovascular imaging for patients with high-risk transient ischemic attack (TIA) or minor stroke in the emergency department (ED) with computed tomography angiography (CTA) of the head and neck is the guideline-recommended standard of care, but it is underutilized in routine practice. We conducted a quality initiative to improve adherence to guidelines.

Methods Between January 2017 and March 2019, we implemented a decision support tool integrated into the electronic ordering system to guide ED physicians to order a CTA on patients with high-risk TIA or minor stroke defined as ongoing neurological deficits in the ED or resolved motor or speech deficits in the preceding $48 \mathrm{~h}$. Data were collected retrospectively pre-intervention and prospectively post-intervention. We used an interrupted time-series analysis for the before-after comparison of the use of CTA among patients who met criteria (main process measure) and those who did not meet criteria (balancing measure).

Results Among 861 patients with TIA or minor stroke, the proportion of patients with high-risk events imaged with a CTA in the ED increased from $12.0 \%$ pre-intervention to $77.0 \%$ post-intervention and this shift was sustained over 11 months. CTA use in those without high-risk events increased to a lesser extent (15.3\% versus $42.9 \%)$. The interrupted time-series analysis showed a step change immediately post-intervention where the increase in CTA use in patients with high-risk events was $51.7 \%$ higher than its use in those without high-risk events $(p<0.001)$. Compared to pre-intervention, the median ED length of stay increased by $2 \mathrm{~h}$ and neurology consultation in the ED was more frequent (5.8\% versus $19.5 \%)$ post-intervention. Conclusion We provide a detailed framework that improved adherence to acute imaging guidelines for patients with TIA or minor stroke and anticipate that our approach could improve acute imaging for such patients in most EDs.
\end{abstract}

Keywords Quality improvement · Transient ischemic attack · Stroke · Imaging

\section{Résumé}

Contexte L'imagerie neurovasculaire pour les patients présentant un risque élevé d'accident ischémique transitoire (AIT) ou d'accident vasculaire cérébral mineur aux services d'urgence, avec une angiographie par tomodensitométrie (CTA) de la tête et du cou, est la norme de soins recommandée par les directives, mais elle est sous-utilisée dans la pratique courante. Nous avons mené une initiative de qualité pour améliorer le respect des lignes directrices.

Amy Y. X. Yu

amyyx.yu@utoronto.ca

1 Hurvitz Brain Sciences Program, Sunnybrook Research Institute, University of Toronto, Toronto, ON, Canada

2 Department of Medicine (Emergency Medicine), University of Toronto, Sunnybrook Health Sciences Centre, Toronto, ON, Canada

3 Department of Medicine (Neurology), University of Toronto, Toronto Western Hospital, Toronto, ON, Canada
4 Department of Medicine (General Internal Medicine), University of Toronto, Toronto General Hospital, Toronto, ON, Canada

5 Department of Medical Imaging, University of Toronto, Sunnybrook Health Sciences Centre, Toronto, ON, Canada

6 ICES, Toronto, ON, Canada

7 Department of Medicine (Neurology), University of Toronto, Sunnybrook Health Sciences Centre, Toronto, ON, Canada 
Méthodes Entre janvier 2017 et mars 2019, nous avons mis en place un outil d'aide à la décision intégré au système de commande électronique pour guider les médecins du service d'urgence à prescrire un CTA sur des patients atteints d'un AIT à haut risque ou d'un AVC mineur défini comme des déficits neurologiques en cours au service des urgences ou une résolution de la motricité ou des troubles de la parole dans les 48 heures précédentes. Les données ont été recueillies rétrospectivement avant l'intervention et prospectivement après l'intervention. Nous avons utilisé une analyse de séries chronologiques interrompues pour la comparaison avant-après de l'utilisation du CTA chez les patients qui répondaient aux critères (mesure principale du processus) et ceux qui ne répondaient pas aux critères (mesure d'équilibrage).

Résultats Parmi les 861 patients atteints d'un AIT ou d'un AVC mineur, la proportion de patients présentant des événements à haut risque imagés avec un CTA au service d'urgence est passé de 12,0\% avant l'intervention à 77,0 \% après l'intervention et ce changement s'est maintenu pendant 11 mois. L'utilisation de CTA chez les personnes sans événements à haut risque a augmenté dans une moindre mesure $(15,3 \%$ contre $42,9 \%)$. L'analyse des séries chronologiques interrompues a montré un changement d'étape immédiatement après l'intervention où l'augmentation de l'utilisation du CTA chez les patients présentant des événements à haut risque était $51,7 \%$ plus élevée que son utilisation chez ceux sans événements à haut risque $(\mathrm{p}<$ 0,001). Par rapport à la pré-intervention, la durée médiane du séjour au SU a augmenté de deux heures et les consultations de neurologie au SU étaient plus fréquentes $(5,8 \%$ contre $19,5 \%)$ après l'intervention.

Conclusion Nous fournissons un cadre détaillé qui a amélioré le respect des lignes directrices en matière d'imagerie aiguë pour les patients souffrant d'AIT ou d'AVC mineur et nous prévoyons que notre approche pourrait améliorer l'imagerie aiguë pour ces patients dans la plupart des urgences.

\section{Clinician's capsule}

What is known about the topic?

Adherence to neurovascular imaging guidelines for patients with high-risk TIA or minor stroke is low in the ED.

\section{What did this study ask?}

Could a multidisciplinary quality improvement initiative improve adherence to imaging guidelines for highrisk TIA or minor stroke in the ED?

\section{What did this study find?}

The use of CTA in patients with high-risk TIA or minor stroke increased from $12.0 \%$ pre-intervention to $77.0 \%$ post-intervention.

\section{Why does this study matter to clinicians?}

Our detailed quality improvement framework should improve the use of appropriate neurovascular imaging in most EDs.

\section{Introduction}

Most patients with ischemic stroke initially present with mild or transient symptoms [1], but transient ischemic attacks (TIA) and minor strokes do not have benign outcomes [2, 3]. Urgent vascular imaging for patients with high-risk TIA or minor stroke is recommended, because the presence of vascular abnormality is associated with higher risk of disability and stroke recurrence $[3,4]$ and guides secondary stroke prevention treatment $[5,6]$. The Canadian Stroke Best Practice Guidelines define a high-risk minor ischemic cerebrovascular event as any ongoing neurological symptoms in the emergency department (ED) or resolved symptoms that included motor or speech deficits in the preceding $48 \mathrm{~h}$ [7].

However, vascular imaging is underutilized in routine clinical practice $[8,9]$. Recent data from the US showed that only $43 \%$ of patients with TIA had vascular imaging with carotid Dopplers or computed tomography angiography (CTA) in the ED [10]. Furthermore, CTA is preferred over carotid Dopplers, because CTA allows for the additional assessment of the intracranial and posterior circulation vessels $[7,11,12]$. Urgent CTA in all patients with suspected minor ischemic cerebrovascular events can negatively impact workflow in the emergency and radiology departments, but a delay in obtaining this test in patients with high-risk events may lead to missed opportunities for stroke treatment and prevention [5].

At our institution, most patients with suspected TIA or minor stroke had a non-contrast CT head in the ED, followed by outpatient carotid Dopplers. The use of CTA was estimated to be 5\% based on clinical practice patterns. We used a multidisciplinary quality improvement initiative involving the neurology, radiology, and emergency departments to increase the use of CTA in patients with suspected high-risk TIA or minor stroke in the ED to a target of $80 \%$ within 11 months. This target was chosen because we recognize that some patients may have contraindications to CTA or decline this test, and investigations may need to be individualized to specific clinical scenarios."

\section{Methods}

\section{Population and setting}

We conducted a quality improvement study at a universityaffiliated comprehensive stroke center with approximately 
800 acute stroke admissions and 60,000 ED visits annually. At baseline, the standard practice for patients with suspected TIA or minor stroke was for ED physicians to order a noncontrast CT head in the ED, arrange outpatient carotid Dopplers within 24-72 h, and send a referral to the stroke prevention clinic. Following in-person meetings with the heads of the neurology, radiology, and emergency departments or their delegates (physicians involved with the department's operations), we identified knowledge gaps on the relative value of CTA as compared to Doppler ultrasound, lack of clarity on which events are high-risk, worries about overwhelming radiology resources from indiscriminate ordering of CTA, and perceived challenges in having CTAs approved and protocolled rapidly, a common issue encountered in the general ED care [13]. We focused on our intervention on these issues.

\section{Intervention}

First, the neurology, radiology, and emergency departments collaboratively established that patients with suspected highrisk TIA or minor stroke and no contraindication to intravenous contrast, at the discretion of the ordering physician, would meet evidence-based criteria for having a CTA in the ED. Second, to facilitate knowledge translation and standardize care, we designed and implemented an electronic decision support tool to guide ED physicians at the time of test ordering as to which patients would benefit from CTA in the ED by prompting the physician to answer two questions: (1) 'Does this patient have any persistent neurological deficits in the ED?' and (2) 'If deficits have resolved, did the patient have motor and/or speech symptoms within the last $48 \mathrm{~h}$ ?' The decision support tool proposed changing the order from non-contrast CT to CTA of the head and neck with a positive answer to either questions in the absence of contraindications to CTA. This suggestion could be manually overridden by the physician. We performed user acceptance testing, by asking front-line ED physicians to read a clinical scenario, complete the questions with pen and paper, and answer whether a CTA was recommended. Third, we provided education for staff physicians in the emergency, neurology, and radiology departments through formal presentations at department rounds, internal communication by the head of each department, and this information was added to the ED physician orientation manual for reference. Fourth, to streamline workflow for the ED physician at the time of imaging ordering, a memorandum of understanding was sent to members of all three departments and posted in the ED.

\section{Timeline}

In the pre-intervention period (January 1st 2017 to October 31st 2017), we identified patients using the stroke prevention clinic's database and data were collected retrospectively. The wash-out period during which the quality improvement initiatives were being planned was between November 2017 and April 2018. The electronic decision support tool was officially launched on May 3rd 2018. For each week during the post-intervention period (May 3rd 2018 to March 31st 2019), data were collected prospectively using active daily review of use of CTA in all patients with suspected TIA or minor stroke admitted to hospital or referred to the Stroke Prevention Clinic. Research assistants screened all referrals and admissions. We excluded patients with intracerebral hemorrhage, and those who were evaluated by the stroke team under the acute "code stroke" protocol, even if the symptoms of stroke were mild or resolving, because the local practice is to image all patients seen as "code stroke" with CT, CTA, and CT perfusion.

\section{Intervention reinforcement}

We used a run chart to monitor the proportion of patients with suspected high-risk TIA or minor stroke who had a CTA in the ED and those who had a CTA without meeting the criteria, the main process and balancing measures [14, 15]. In the first 15 weeks post-intervention, we reinforced the intervention through daily active surveillance and contacting the physicians involved in protocol deviations via email to ensure they were aware of the protocol, knew where to find its details, and discuss any case-specific ambiguities.

\section{Secondary measures}

Secondary balancing measures included the proportion of clinically relevant findings on CTA, the ED length of stay, and the proportion of patients who had a neurology consultation in the ED. Clinically relevant CTA findings were defined as the presence of symptomatic intracranial vessel disease (occlusion or $\geq 50 \%$ stenosis), symptomatic extracranial vessel disease (occlusion or $\geq 50 \%$ stenosis or stenosis $<50 \%$ with ulcerated plaque or thrombus), or other findings relevant to the symptoms (e.g., dissection, multifocal vessel irregularity, moyamoya). CTA findings were abstracted by trained abstractors and verified by a stroke neurologist (AYXY). We also evaluated clinical outcomes by linking our data to the provincial Discharge Abstract Database and the Ontario Registered Persons Database housed at ICES (previously called the Institute for Clinical Evaluative Sciences) to determine all-cause hospital readmissions, and re-admission for stroke at 90 days, identified using validated International Classification of Diseases 10th Canadian Iteration codes H34.1, I63.x, I64.x, I61.x [16]. The datasets were linked using unique encoded identifiers and analyzed at ICES. 
Table 1 Baseline patient characteristics

\begin{tabular}{|c|c|c|c|}
\hline & $\begin{array}{l}\text { Pre-intervention } \\
(N=344)\end{array}$ & $\begin{array}{l}\text { Post-intervention } \\
(N=502)\end{array}$ & $p$ value \\
\hline Median age (IQR) in years & $69(58,80)$ & $68(57,80)$ & 0.83 \\
\hline Female $n(\%)$ & $210(61.0 \%)$ & $274(54.6 \%)$ & 0.06 \\
\hline High-risk event ${ }^{\mathrm{a}} n(\%)$ & $233(67.7 \%)$ & $339(67.5 \%)$ & 0.95 \\
\hline Ongoing symptoms n (\%) & $142(41.3 \%)$ & $189(37.6 \%)$ & 0.29 \\
\hline $\begin{array}{l}\text { Resolved symptoms with motor or speech } \\
\text { involvement in last } 48 \mathrm{~h} \mathrm{n} \mathrm{( \% )}\end{array}$ & $91(26.5 \%)$ & $150(29.9 \%)$ & 0.28 \\
\hline \multicolumn{4}{|l|}{ Comorbidities $n(\%)$} \\
\hline Transient ischemic attack & $30(8.7 \%)$ & $41(8.2 \%)$ & 0.78 \\
\hline Stroke & $40(11.6 \%)$ & $62(12.4 \%)$ & 0.75 \\
\hline Hypertension & $185(53.8 \%)$ & $270(53.8 \%)$ & 0.99 \\
\hline Congestive heart failure & $7(2.0 \%)$ & $28(5.6 \%)$ & 0.01 \\
\hline Coronary artery disease & $40(11.6 \%)$ & $73(14.5 \%)$ & 0.22 \\
\hline Diabetes & $55(16.0 \%)$ & $103(20.5 \%)$ & 0.1 \\
\hline Dyslipidemia & $172(50.0 \%)$ & $219(43.6 \%)$ & 0.07 \\
\hline Atrial fibrillation & $37(10.8 \%)$ & $47(9.4 \%)$ & 0.51 \\
\hline Smoking & $32(9.3 \%)$ & $33(6.6 \%)$ & 0.14 \\
\hline
\end{tabular}

$I Q R$ interquartile range

${ }^{a}$ Defined as ongoing neurological symptoms in the emergency department OR resolved symptoms that included motor or speech deficits in the last $48 \mathrm{~h}$

\section{Research ethics}

This study was approved by the Sunnybrook Health Sciences Centre Research Ethics Board with a waiver of individual patient consent.

\section{Statistical methods}

Patient characteristics pre- and post-intervention were compared using $t$ tests (continuous variables) and Chi-square tests (categorical variables). We used an interrupted timeseries analysis with multiple group comparisons to determine whether there was a change in the rates of CTA use patients who met high-risk criteria versus those who did not, pre- and post-intervention, beyond what would be expected from the pre-intervention temporal trends [17]. For this analysis, time periods were categorized into 4 -week intervals to create more stable estimates when calculating the rates of CTA. We compared the difference in CTA utilization between patients with and without high-risk events by testing the difference in the change in slope between these two patient groups during the pre-intervention phase $\left(\beta_{5}\right)$ and post-intervention phase $\left(\beta_{7}\right)$, as well as testing the difference in step change between these two patient groups immediately post-intervention $\left(\beta_{6}\right)$. We plotted residuals over time and used the Durban-Watson statistic to test for autocorrelation. Statistical significance was defined as $p<0.05$. Analyses were performed using SAS 9.4 (SAS Institute Inc., Cary,
NC, USA) and figures were generated using Excel (Microsoft Office 2016).

\section{Results}

We identified 861 patients with TIA or minor stroke during the study period. We successfully linked 846 patients ( $n=344$ pre-intervention, $n=502$ post-intervention) to administrative data. The patients who could not be linked $(1.7 \%, n=15)$ were included in the run chart and time-series analyses, but they were not included in other analyses.

Patient characteristics were similar pre- and post-intervention (Table 1). Overall, ED CTA use increased from 13.1 to $65.9 \%$, equivalent to about six CTAs per week (Table 2). The proportion of patients with high-risk events undergoing a CTA in the ED increased from $12.0 \%$ (28/233) preintervention to $77.0 \%(261 / 229)$ post-intervention. The proportion of patients who did not have high-risk events undergoing a CTA in the ED increased from 15.3\% (17/111) pre-intervention to $42.9 \%$ (70/163) post-intervention.

We showed in Fig. 1 the run chart of frequency of CTA use in the ED, stratified by whether the event was clinically high risk or not. For patients with high-risk TIA/minor stroke, there was an upward shift in CTA use post- compared to pre-intervention. No trends were observed preintervention. Early post-intervention, there was an upward trend between weeks 3 and 11, after which only four data 
Table 2 Care and outcomes pre- and post-intervention

\begin{tabular}{|c|c|c|c|}
\hline & Pre-intervention $(N=344)$ & $\begin{array}{l}\text { Post-intervention } \\
(N=502)\end{array}$ & $p$ value \\
\hline Non-contrast CT in ED $n(\%)$ & $329(95.6 \%)$ & $494(98.4 \%)$ & 0.02 \\
\hline CTA in ED $n(\%)$ & $45(13.1 \%)$ & $331(65.9 \%)$ & $<0.0001$ \\
\hline Carotid Doppler ordered by ED $n(\%)$ & $321(93.3 \%)$ & $178(35.5 \%)$ & $<0.0001$ \\
\hline CTA ordered from clinic $n(\%)$ & $79(23.0 \%)$ & $30(6.0 \%)$ & $<0.0001$ \\
\hline Both carotid Doppler and CTA $n(\%)$ & $79(23.0 \%)$ & $29(5.8 \%)$ & $<0.0001$ \\
\hline Median days (IQR) to first intracranial vascular imaging & 11 days $(0,22)$ & 0 day $(0,0)$ & $<0.0001$ \\
\hline ED neurology consult, $n(\%)$ & $20(5.8 \%)$ & $98(19.5 \%)$ & $<.0001$ \\
\hline Discharge from ED with dual antiplatelet treatment & $11(3.2 \%)$ & $40(8.0 \%)$ & 0.004 \\
\hline All-cause re-admission & $31(9.0 \%)$ & $54(10.8 \%)$ & 0.41 \\
\hline Stroke re-admission & $6(1.7 \%)$ & $12(2.4 \%)$ & 0.52 \\
\hline
\end{tabular}

$E D$ emergency department, $C T$ computed tomography, $C T A$ computed tomography angiography, $I Q R$ interquartile range

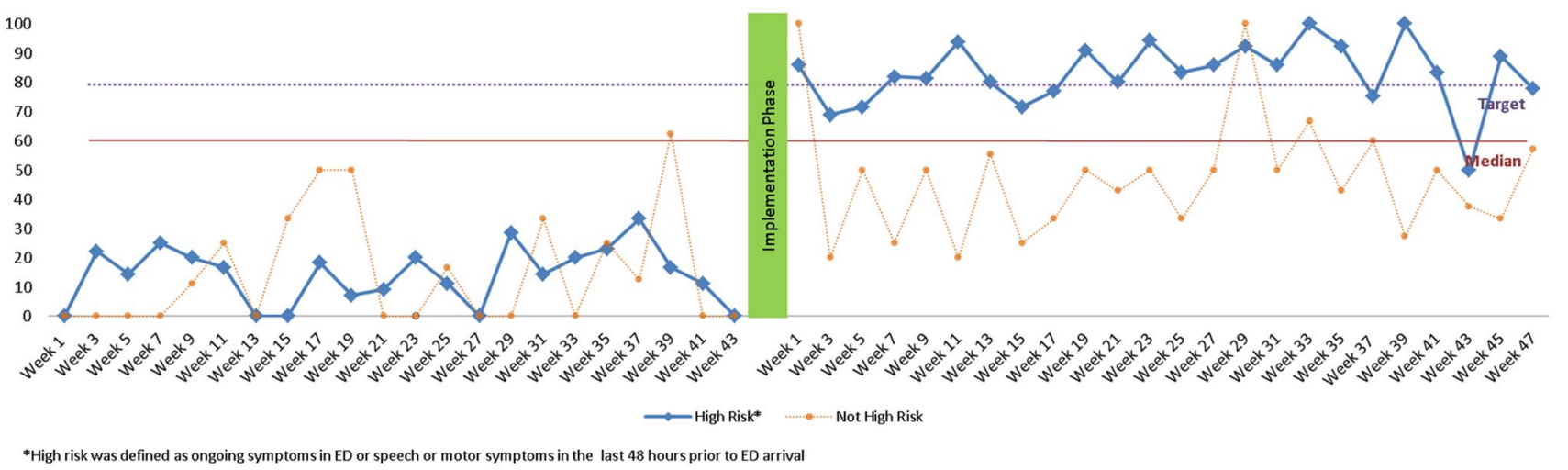

Fig. 1 Run chart of the use of computed tomography angiography for patients with TIA or minor stroke in the emergency department. Red line indicates the median CTA use over the entire study period (pre- and post-intervention). Dotted purple line indicates our target. We aimed for $80 \%$ of patients with high-risk event undergoing CTA in the emergency department points were below our target and there was no downward trend. There were three runs: the first run ended when the intervention was implemented, and the second run ended at week 43 (at outlier data point). On the other hand, in those without a high-risk event, there were no shifts above the median and no trends. Thus, the uptake of the intervention to increase CTA use for high-risk event occurred early and remained high and the intervention affected those without high-risk event to a lesser extent. There were two outlier data points (week 43 for patients with high-risk event and week 29 for those without high-risk event). We are not aware of any events during these weeks to affect the intervention and, particularly for the outlier data point at week 29 , we were reassured that there were no subsequent trends or shift.

The interrupted time-series analysis showed no difference in the slope of CTA use in patients with or without high-risk events pre- $\left(\beta_{5}=-2.30, p=0.11\right)$ and post-intervention $\left(\beta_{7}=2.29, p=0.22\right)$. These estimates can be interpreted as there was only a $2.3 \%$ difference in the slope change of CTA use when comparing patients with high-risk events to those without. However, there was a large immediate post-intervention step change where the increase in CTA use in patients with high-risk events was $51.7 \%$ higher than in those without high-risk events and this was statistically significant $\left(\beta_{6}=51.67, p<0.001\right)$. Thus, the increase in CTA use occurred immediately post-intervention and was largely in people with high-risk events.

We identified a clinically relevant abnormality in $20.0 \%$ (9/45) of all CTA done in the pre-intervention period compared to $16.3 \%(54 / 331)$ in the post-intervention period. Post-intervention, a higher proportion of patients had an urgent neurology consultation or were discharged from the ED with dual antiplatelet therapy compared to pre-intervention (Table 2). 
Table 3 Median ED length of stay (IQR) in hours pre- and post-intervention

\begin{tabular}{llll}
\hline & Pre-intervention & Post-intervention & $P$ value \\
\hline Overall cohort & $6.3(5.0,8.2)$ & $8.1(6.6,10.3)$ & $<.0001$ \\
& $n=344$ & $n=502$ & \\
Patients without ED CTA & $6.2(5.0,8.0)$ & $7.3(5.7,8.8)$ & $<.0001$ \\
& $n=299$ & $n=171$ \\
Patients with ED CTA & $8.6(6.1,10.6)$ & $8.7(6.9,10.7)$ & 0.30 \\
Patients with ED CTA and high-risk event & $n=45$ & $n=331$ & \\
& $9.4(6.2,11.4)$ & $8.7(7.1,10.8)$ & 0.83 \\
Patients with ED CTA without high-risk event & $n=28$ & $n=261$ & \\
& $7.7(5.9,8.8)$ & $9.0(6.6,10.5)$ & 0.07 \\
\end{tabular}

$E D$ emergency department, $C T$ computed tomography, CTA computed tomography angiography, IQR: interquartile range
A notable benefit of our intervention was a reduction in duplicate vascular imaging (Table 2). In the pre-intervention period, $23.0 \%$ of patients underwent both carotid Dopplers, ordered from the ED, and a subsequent CTA, ordered by the neurologist from the stroke prevention clinic for the same clinical event. Post-intervention, only $5.8 \%$ of the patients had both tests. Further, the median time to first intracranial vascular imaging reduced from 11 days [22] pre-intervention to 0 day $(0,0)$ post-intervention. All-cause hospital re-admission and re-admission for stroke within 90 days were overall infrequent and were similar pre- and post-intervention (Table 2).

We found that the median ED length of stay increased post- compared to pre-intervention and that patients with CTA in the ED had longer stay than those who did not in the overall cohort, regardless of the event risk (Table 3). Compared to pre-intervention, the length of stay postintervention was stable in patients with a CTA, but increased by an hour in patients who did not have a CTA.

\section{Discussion}

\section{Summary}

We successfully implemented a multidisciplinary and multifaceted quality improvement initiative and increased the use of CTA in the target population, improved rapid access to CTA, and reduced waste associated with duplicate vascular imaging. The interrupted time-series analysis showed a statistically significant increase in CTA use immediately post-intervention that is not explained by baseline temporal trends and mainly seen in patient with high-risk events. The number of re-admissions was overall low, consistent with other reports, and we were underpowered to detect differences in these clinical outcomes $[18,19]$.

\section{Strengths and lessons}

The strengths of this project include its multidisciplinary approach involving three departments and the implementation of a decision tool integrated into the electronic health system to guide physicians at the time of test ordering. These steps enabled our group to focus ED and radiology resources on patients with high-risk TIA or minor stroke. Although the ED length of stay was overall longer among patients imaged with CTA compared to those who were not, ED stay increased post- compared to pre-intervention in patients who did not have CTA while it remained stable among those who had a CTA, suggesting there are factors external to our interventions that are prolonging stay, such as increasing ED volumes and crowding metrics [20]. We found that $16.3 \%$ of the CTAs done during the post-intervention period had a clinically significant abnormality and more neurology consultations were completed in the ED. More work on using different clinical triaging tools to select high-risk patients who may benefit from CTA [21], or neurology consultation in the ED, and further effort to improve patient flow in the ED for patients with TIA or minor stroke are needed.

\section{Limitations}

Our study has several limitations worth discussing. First, we were not able to directly measure the uptake of the electronic decision tool by ED physicians. However, the daily active surveillance allowed us to track the success of the overall quality improvement project. The effectiveness of including a risk-stratification tool at the time of physician order entry has been previously shown in the context of prescriptions for venous thromboembolism prophylaxis [22]. We are not aware of any external factors that could have contributed to the improved access to CTA during the study 
period, but cannot exclude any residual confounding. Second, although our intervention was designed to increase the use of CTA in patients with high-risk event, an increase in CTA use was nevertheless seen in those without high-risk event. We nevertheless acknowledge that a CTA may be necessary for some patients who did not meet the criteria in our intervention. For example, it may be appropriate in patients with symptoms suggestive of posterior circulation TIA even in the absence of motor or speech symptoms. We could not capture this level of clinical judgment through chart reviews. Third, patients discharged from the ED who were not referred to our institution's stroke prevention clinic or who were admitted without consulting the neurology team could not be captured, but this is not the usual practice at our institution. Most patients with suspected TIA or minor stroke would have been identified with our active surveillance method. Fourth, although our study was not designed to study the safety of CTA, we did not identify any adverse events during the study period. The safety of CTA in stroke care has been reported elsewhere [23, 24]. Finally, given this is a single-centre study, future work is needed to assess the generalizability of our approach in other settings.

\section{Implications for research or practice}

Although many studies have identified variability in the provision of acute vascular imaging in the ED [8, 10, 18], most quality improvement initiatives to date have aimed to develop referral pathways to support primary care physicians [19] or to improve access or quality of outpatient secondary stroke prevention care [25-27]. Few have sought to optimize care in the ED. Yet, urgent vascular imaging is necessary to identify patients who may benefit from carotid revascularization [28], dual antiplatelet therapy [29], and even potentially acute revascularization procedures $[6,30$, 31]. A large population-based Canadian study found that patients with TIA or minor stroke in EDs with higher use of CTA had lower hospital re-admissions in the following 90 days than those who were treated in EDs with lower use of CTA [18]. It is therefore relevant to optimize adherence to guideline recommendations for acute vascular imaging. Our project provides a framework that can be easily adaptable to other centers and used to either optimize the use of CTA imaging for patients with TIA or minor stroke or to reduce unnecessary CTA.

\section{Conclusion}

A multidisciplinary quality improvement intervention involving an electronic decision tool improved adherence to acute imaging guidelines in the evaluation of people with
TIA or minor stroke. We provide a detailed work plan that can be applied and adapted to improve imaging in most EDs.

Acknowledgements This study was supported by ICES, formally Institute for Clinical Evaluative Sciences, which is funded by an annual grant from the Ontario Ministry of Health and Long-Term Care (MOHLTC). The opinions, results and conclusions reported in this paper are those of the authors and are independent from the funding sources. No endorsement by ICES or the Ontario MOHLTC is intended or should be inferred. Parts of this material are based on data and/or information compiled and provided by CIHI. However, the analyses, conclusions, opinions and statements expressed in the material are those of the author(s), and not necessarily those of CIHI.

Author contributions AK: study design, data collection, analysis, drafting manuscript, AV: study design, data collection, analysis, drafting manuscript, IJK: data collection, reviewing3manuscript, NK: data collection, reviewing manuscript, KS: data collection, reviewing manuscript, LKC: study design, reviewing manuscript, MKK: study design, reviewing manuscript, JF: analysis, reviewing manuscript, SS: study design, reviewing manuscript, RHS: study design, reviewing manuscript, AYXY: study design, data collection, analysis, drafting manuscript, funding.

Funding This study is funded by a grant from the Academic Health Sciences Centres of Ontario Alternative Funding Plan Innovation Fund. AYXY is supported by a New Investigator Award and the HJ Barnett Award from Heart and Stroke. RHS is supported by a clinician-scientist phase II award from the Heart and Stroke Foundation of Canada. MKK is supported by a mid-career investigator award from the Heart and Stroke Foundation of Canada and holds the Lillian Love Chair in Women's Health at the University Health Network.

Code availability Not applicable.

\section{Declarations}

Conflict of interest The authors report no disclosure related to this study.

Ethics approval This study was approved by the Sunnybrook Health Sciences Centre Research Ethics Board with a waiver of individual patient consent.

\section{References}

1. Reeves M, Khoury J, Alwell K, Moomaw C, Flaherty M, Woo D, et al. Distribution of National Institutes of Health stroke scale in the Cincinnati/Northern Kentucky Stroke Study. Stroke J Cereb Circ. 2013;44(11):3211-3.

2. Smith EE, Fonarow GC, Reeves MJ, Cox M, Olson DM, Hernandez AF, et al. Outcomes in mild or rapidly improving stroke not treated with intravenous recombinant tissue-type plasminogen activator: findings from Get With The Guidelines-Stroke. Stroke J Cereb Circ. 2011;42(11):3110-5.

3. Coutts SB, Modi J, Patel SK, Aram H, Demchuk AM, Goyal $\mathrm{M}$, et al. What causes disability after transient ischemic attack and minor stroke?: Results from the CT and MRI in the Triage of TIA and minor Cerebrovascular Events to Identify High Risk Patients (CATCH) Study. Stroke J Cereb Circ. 2012;43(11):3018-22. 
4. Wasserman J, Perry J, Dowlatshahi D, Stotts G, Stiell I, Sutherland J, et al. Stratified, urgent care for transient ischemic attack results in low stroke rates. Stroke J Cereb Circ. 2010;41(11):2601-5.

5. Graham BR, Menon BK, Coutts SB, Goyal M, Demchuk AM. Computed tomographic angiography in stroke and high-risk transient ischemic attack: Do not leave the emergency department without it! Int J Stroke. 2018;13(7):673-86.

6. Yu AY, Hill MD, Coutts SB. Should minor stroke patients be thrombolyzed? A focused review and future directions. Int $\mathbf{J}$ Stroke. 2015;10(3):292-7.

7. Wein T, Lindsay MP, Cote R, Foley N, Berlingieri J, Bhogal S, et al. Canadian stroke best practice recommendations: Secondary prevention of stroke, sixth edition practice guidelines, update 2017. Int J Stroke. 2017:1747493017743062.

8. Ranta A, Barber PA. Transient ischemic attack service provision: a review of available service models. Neurology. 2016;86(10):947-53.

9. Kapral MK, Hall R, Fang J, Austin PC, Silver FL, Gladstone DJ, et al. Association between hospitalization and care after transient ischemic attack or minor stroke. Neurology. 2016;86(17):1582-9.

10. Timpone VM, Jensen A, Poisson SN, Trivedi PS. Compliance with imaging guidelines for workup of transient ischemic attack: evidence from the nationwide emergency department sample. Stroke. 2020;51(8):2563-7.

11. Wintermark M, Sanelli PC, Albers GW, Bello JA, Derdeyn CP, Hetts SW, et al. Imaging recommendations for acute stroke and transient ischemic attack patients: a joint statement by the American Society of Neuroradiology, the American College of Radiology and the Society of NeuroInterventional Surgery. J Am College Radiol. 2013;10(11):828-32.

12. Easton JD, Saver JL, Albers GW, Alberts MJ, Chaturvedi S, Feldmann E, et al. Definition and evaluation of transient ischemic attack: a scientific statement for healthcare professionals from the American Heart Association/American Stroke Association Stroke Council; Council on Cardiovascular Surgery and Anesthesia; Council on Cardiovascular Radiology and Intervention; Council on Cardiovascular Nursing; and the Interdisciplinary Council on Peripheral Vascular Disease. The American Academy of Neurology affirms the value of this statement as an educational tool for neurologists. Stroke; a journal of cerebral circulation. 2009;40(6):2276-93.

13. Bhayana R, Wang CD, Menezes RJ, Bartlett ES, Choi J. Optimising after-hours workflow of computed tomography orders in the emergency department. BMJ open quality. 2020;9(3).

14. Perla RJ, Provost LP, Murray SK. The run chart: a simple analytical tool for learning from variation in healthcare processes. BMJ Qual Saf. 2011;20(1):46-51.

15. Taylor MJ, McNicholas C, Nicolay C, Darzi A, Bell D, Reed JE. Systematic review of the application of the plan-do-studyact method to improve quality in healthcare. BMJ Qual Saf. 2014;23(4):290-8.

16. Kokotailo RA, Hill MD. Coding of stroke and stroke risk factors using international classification of diseases, revisions 9 and 10. Stroke J Cereb Circ. 2005;36(8):1776-81.

17. Linden A. Using group-based trajectory modelling to enhance causal inference in interrupted time series analysis. J Eval Clin Pract. 2018;24(3):502-7.
18. Leong M, Stang JM, McGuire N, Lang E, Coutts SB, Patocka C. Regional variation in transient ischemic attack and minor stroke in alberta emergency departments. Stroke. 2020;51(6):1820-4.

19. Ranta A, Dovey S, Weatherall M, O'Dea D, Gommans J, Tilyard M. Cluster randomized controlled trial of TIA electronic decision support in primary care. Neurology. 2015;84(15):1545-51.

20. Rowe BH, McRae A, Rosychuk RJ. Temporal trends in emergency department volumes and crowding metrics in a western Canadian province: a population-based, administrative data study. BMC Health Serv Res. 2020;20(1):356.

21. Perry JJ, Sivilotti MLA, Émond M, Stiell IG, Stotts G, Lee J, et al. Prospective validation of Canadian TIA Score and comparison with $\mathrm{ABCD} 2$ and $\mathrm{ABCD} 2 \mathrm{i}$ for subsequent stroke risk after transient ischaemic attack: multicentre prospective cohort study. BMJ. 2021;372:n49.

22. Preston H, Swan I, Davies L, Dummer S, Aravindan V, Beh YY, et al. Improving VTE risk assessment and prophylaxis prescribing rate in medical patients: integrating risk assessment tool into the workflow. BMJ open quality. 2020;9(2).

23. Becker BA, Yeich T, Jaffe JT, Sun S, Chen Y, Rebert T, et al. Impact of creatinine screening on contrast-induced nephropathy following computerized tomography for stroke. Am J Emerg Med. 2020.

24. Hopyan JJ, Gladstone DJ, Mallia G, Schiff J, Fox AJ, Symons SP, et al. Renal safety of CT angiography and perfusion imaging in the emergency evaluation of acute stroke. Am J Neuroradiol. 2008;29(10):1826-30.

25. Rothwell PM, Giles MF, Chandratheva A, Marquardt L, Geraghty O, Redgrave JN, et al. Effect of urgent treatment of transient ischaemic attack and minor stroke on early recurrent stroke (EXPRESS study): a prospective population-based sequential comparison. Lancet. 2007;370(9596):1432-42.

26. Lavallee PC, Meseguer E, Abboud H, Cabrejo L, Olivot JM, Simon O, et al. A transient ischaemic attack clinic with round-theclock access (SOS-TIA): feasibility and effects. Lancet Neurol. 2007;6(11):953-60.

27. Duncan PW, Bushnell CD, Rosamond WD, Jones Berkeley SB, Gesell SB, D'Agostino RB Jr, et al. The Comprehensive PostAcute Stroke Services (COMPASS) study: design and methods for a cluster-randomized pragmatic trial. BMC Neurol. 2017;17(1):133.

28. Rothwell PM, Eliasziw M, Gutnikov SA, Warlow CP, Barnett HJ. Carotid Endarterectomy Trialists C. Endarterectomy for symptomatic carotid stenosis in relation to clinical subgroups and timing of surgery. Lancet. 2004;363(9413):915-24.

29. Chimowitz MI, Lynn MJ, Derdeyn CP, Turan TN, Fiorella D, Lane $\mathrm{BF}$, et al. Stenting versus aggressive medical therapy for intracranial arterial stenosis. N Engl J Med. 2011;365(11):993-1003.

30. Coutts SB, Dubuc V, Mandzia J, Kenney C, Demchuk AM, Smith EE, et al. Tenecteplase-tissue-type plasminogen activator evaluation for minor ischemic stroke with proven occlusion. Stroke. 2015;46(3):769-74.

31. Seners P, Ben Hassen W, Lapergue B, Arquizan C, Heldner MR, Henon H, et al. Prediction of Early Neurological Deterioration in Individuals With Minor Stroke and Large Vessel Occlusion Intended for Intravenous Thrombolysis Alone. JAMA Neurol. 2021. 